\title{
14
}

\section{Analysis of questionnaire results}

\section{P. Bernus and L. Nemes}

The conclusions below are drawn from a review of the answers given to each of the three Questionnaires by the Purdue, CIMOSA and GRAI-GIM teams.

\subsection{ARCHITECTURE}

\subsubsection{Users of the architecture}

The architectures are targeted at diverse audience, such as

1. Consultants, Engineering Firms

2. Designers of the enterprise (technical level)

3. Decision makers involved in the design and development of the enterprise such as:

(a) Projects to integrate enterprise activities (carried out as a project within the enterprise

(b) Commissioned projects (e.g., for the delivery of a subsystem to the enterprise such as a manufacturing system or any other customer service.)

Note: generic architectures are to be handled by many decision makers who might be at various levels in the decision making hierarchy.

\subsubsection{Intended scope of the architecture}

We have asked what area of enterprise activity is generally involved in generic architecture proposals. The answer to this question is twofold:

- What areas of activity are intended to be covered

- What areas have already been covered and to what extent have they been covered. 
In other words we wanted to determine the potential of the architecture versus the actual state of its use as of today.

The first question is, in a way, more important to answer than the second but watching closely

- What is the time frame in which the missing details can in fact be delivered.

- Are the methods already provided with the architecture satisfactory enough so that we can expect that the not yet covered areas will indeed be tackled in the same quality as the already worked out details have been.

As a result of these ponderables the following conclusions can be drawn.

- The following areas of enterprise activity are common to all the architectures:

Product distribution, production planning, manufacturing control, manufacturing, material handling, storage and transport, testing and quality control, logistics, acquisition, information technology infrastructure, (including computer, communications) computing, database management systems, etc.

- Those areas which are common to some but not all:

Product design, infrastructure, communications (excluding computer communications), education, strategic enterprise management, resource management, factory development (i.e. development, of the production facility), factory design, factory maintenance.

- Listed only by Purdue:

Legal services, market research.

- Listed only by CIMOSA:

Product research and development, factory building and modernization, infrastructure, energy, infrastructure, buildings and grounds.

- Listed only by GRAI-GIM:

Product management, marketing function, project management.

- These items not covered by any of the architectures (or the issue has not yet been addressed):

Customer services, product maintenance, marketing.

Enterprise integration architectures have grown out of manufacturing integration architectures and methodologies, thus their initial scope is the same as for manufacturing integration. The actual expertise and worked out details are in this area.

However, as we shall see later these enterprise approaches look at more aspects even of the manufacturing related activities than the earlier manufacturing integration projects did. 


\subsubsection{What is the highest intended level of genericity on which this architecture is applicable?}

Every type of industrial enterprise can be tackled using these generic architectures, but to date the actual work on details has concentrated on those industries which have the characteristics of discrete parts manufacturing or of continuous process manufacturing (electronic equipment, paper, chemical, food processing, mechanical and electro mechanical part and machine manufacturing, car industry).

It appears, however, that the service industries can also be tackled by these architectures (e.g., engineering firms, software firms, etc.).

\subsubsection{Which stage of systems development is addressed by the archi- tecture?}

By intention all stages of systems development are fully covered, thus requirements, design, implementation (configuration and reconfiguration), operation and maintenance. 'Being covered by the architecture' should mean that the architecture must describe

- generic models for the enterprise (at each level of the development process), and

- generic models of the processes involved in producing the above.

It is a major source of confusion that each of the three architectures uses a different terminology for the above concepts.

The Purdue and GRAI-GIM way of presenting the architecture is based on the engineer's view, (1) what project specific major deliverables are there? and (2) how to produce them?

The CIMOSA way of presenting the architectures is according to an abstract categorization of models.

The modelling methods of these architectures allow the entire life history of the integrated enterprise to be covered.

Generic models for this are available in the form of the CIMOSA life cycle model, or the Purdue Architecture diagram.

\subsubsection{What are the goals which we would like to attain by developing generic CIM architectures? (What are the main factors in indus- try's need?)}

It was felt by the groups answering these questions that the important and essential factors leading to the development of generic architectures are: ${ }^{1}$ 
** Better use of current resources

*** Integration of current technology

** Cutting excessive costs of having to develop enterprise integration systems individually

** Improve the quality of the developed individual enterprises/ manufacturing systems/CIM systems, etc. (goal orientedness)

** Enabling the development of a marketplace for compatible products for integration

* Contain metrics for exploring economic/technological options

* Integration of the decision making process

* Obtain flexibility in system configuration and adaptation to change in requirements

* To develop for people involved the concept of the integrated nature of the business. This includes understanding, awareness, and training.

* Development of a documented form of the enterprise processes. This is an essential factor for assessing and controlling the quality of the process and thereby the quality of the product.

\subsubsection{De facto scope and detail}

\subsubsection{Case studies}

A number of individual case studies have been prepared for both GRAIGIM and CIMOSA. The Purdue architecture has also been applied to individual cases in industry although it is very new, however, the Purdue Reference Model which concentrates on the manufacturing and manufacturing control areas was tested on numerous industry cases. In fact the CIMOSA and GRAI-GIM case studies have also addressed different subparts of the scope.

1. The number of *s show how many have felt that the factor was essential. 


\subsubsection{Generic architectural models}

The generic architectures in the abstract have almost all the enterprise activities in scope. Detailed generic models which can be used as cookbook examples are at the moment not available except for a few cases, such as:

(a) Available

- The Purdue reference model for manufacturing control

- Production Planning and Production Control Models produced by the GRAI method

- Strategic Enterprise Management Models produced by the GRAI method

(b) Under Development

- Computer Aided Enterprise Engineering Model/Environment (a software house/university project of CIMOSA)

- Fiat/Renault generic enterprise requirements/system design/implementation models.

The CIMOSA Integrating Infrastructure is a generic model for the information integrating infrastructure and is intended to be developed using ISO and other standards (such as OSI (Open Systems Interconnection), ODP (Open Distributed Processing) or RDA (Remote Database Access), and user interface standards etc.) Note that various similar projects exist in theU.S., Canada and in the Asia-Pacific region and it is unlikely that any one will become completely dominant.

The importance of these standards is that the existence of a concrete example for a generic standard is essential for industry acceptance.

\subsubsection{Omissions}

- The importance of equal treatment of economic and human aspects has only recently been acknowledged by the more technically oriented methodologies. Today, the intentions of these architectures is to be as complete as possible.

- The economic aspect of enterprise integration has improved in the last 8 years, e.g., the GRAI methodology includes the ECOGRAI method.

- The Purdue architecture explicitly treats the human aspect.

\subsubsection{Interaction of the modelling framework selected and existing standards expressed in different modelling terms}

CIMOSA has adopted a uniform modelling framework and the effect of the above problem is currently being investigated. 
The Purdue architecture does not use a fixed modelling framework, so the problem does not arise.

The GRAI-GIM architecture is based on widely accepted existing modelling methods. Where not (in its detailed conceptual decisional model and the operational model) there are modelling methods which are quasi standard (IDEF0 and Petri nets). Where GRAI adds a new method (GRAI-grid) there are no standards available and it is hoped that one of GRAI's inputs to CIMOSA will be the development of CIMOSA's decisional/organizational aspects.

\subsubsection{Systems extensibility}

In each of the architectures the necessity of extending existing systems has been taken into account.

There are subtle differences, e.g. the Purdue architecture proposes to remodel the existing part of the system only after the new system has been functionally defined to a certain level.

The CIMOSA life-cycle model proposes to fill the modelling framework, using reverse engineering methods, for the existing system.

Proof that the transition from old to new systems can be done using the considered architectures is based on a mixture of theoretical consideration, feasibility studies and repeated industry experience (Purdue, GRAI).

It is therefore important that the life cycle models of CIMOSA and the Purdue Architecture description explicitly give methods to describe this migration strategy. However, a detailed migration strategy to deal with legacy systems (application programs and databases) is yet to be developed for each architecture, especially for migration of mission critical systems.

\subsubsection{Resilience of systems using the generic architectures}

Perhaps the most important message of the evaluation is that changeability (which underlies resilience) is a designed property of the enterprise or any of its subsystems.

A generally accepted structural division, used explicitly in both Purdue and CIMOSA, is the division of the control functions from the controlled functions. This gives the system a general quality of localizing the effects of change. E.g., the same elementary technological processes may be entirely rearranged using a different control system and still the elementary processes may remain the same. This is important given the different phases 
and speed of technological shifts in the computing/control technology and manufacturing technology.

\subsubsection{Skills required to implement}

The architectures appear to be very different from this aspect. This involves the language, style, and presupposed knowledge on behalf of the practitioner.

The Purdue architecture is easily understood by industrial engineers while the CIMOSA and GRAI-GIM architectures need some information technology background for ready understanding.

\subsubsection{Development potential with respect to skills to implement}

CIMOSA intends to finalize the modelling framework in a way that hides from the practitioner the underlying theoretical complexity.

Purdue, when its practical engineering approach is mapped with the GIM or CIMOSA life cycle models, will become theoretically under-pinned and it is expected that the three architectures can be merged.

The electronic industry is more receptive of formal methods using abstract models than is the process industry. It is therefore expected that the application of CIMOSA and GRAI to process industry case studies and the application of the Purdue Architecture to electronic industry case studies will bring out the fundamental correspondences as well as to help identify mutual contribution between them.

\subsubsection{Dependence on new technologies}

It was the opinion of the majority that for enterprise integration architectures to be practicable, there will be an extensive need for a number of new information technologies.

Distributed Databases (relational and heterogeneous), Open Systems Interconnection, Open Distributed Processing, AI Techniques, Fourth Generation Languages were all mentioned as important conditions for practical implementations.

Distributed information technology is a currently changing $\mathrm{R}$ and $\mathrm{D}$ area and too early standardization of higher levels is not likely to be long lived.

There was no one technology mentioned, however, which seems to lock the user of these architectures into the use of that technology alone. 
One strategic question arises concerning the Information Integration Infrastructure (IIS) of CIMOSA. It should be assessed to what extent the specifications of IIS will accommodate (be resilient to) the obvious spread of distributed AI techniques in the area of information integration.

\subsubsection{What is your opinion about the future prospects for this archi- tecture?}

Answers to this question by the developers of the three architectures are hard to evaluate. CIMOSA claims the setting of standards, presumably referring to the rigorous modelling and specification methods. Purdue claims industry acceptance in its present form, backed by the Purdue consortium. GRAI claims a theoretical impact (by introducing the decisional aspect and its modelling methods) and influencing the other two architectures to develop in this direction. Also the ECOGRAI method may evolve to become an influential part of generic architectures and methodologies.

\subsubsection{What has been the main reason for the development of this architecture?}

The Purdue and GRAI Architectures were initially based on academic developments with industry support and utilization by a group of firms.

CIMOSA is a product of industrial research, backed by academia, but the main reason has been for the development of a standard and for utilization by a group of firms.

Today each of the architectures is backed by a group of firms.

\subsubsection{What is the driving force behind this architecture?}

The major reason for the development of generic architectures seems to be technology pull, i.e. on the basis of industrial needs rather than on earlier developed technology being used in a new area of application.

This has created a situation in which rapid, partial development is easily made. It is then very hard to withhold results until they are really finished and under-pinned with a clear theoretical base. The reason for the creation of this Task Force is a symptom of the above situation.

Thus, enterprises utilizing the generic architectures need to understand that they are technologies which are still developing. 
The decision of an enterprise to apply these architectures should be based on strategic decisions because the expected benefits can only be assured if the maturity level of the enterprise is raised to a sufficient extent.

\subsubsection{Adequacy}

The strong points of the CIMOSA architecture are the following:

- It is developing an overall framework supporting engineering and application (i.e. designing and building the enterprise) called the Information Integration Infrastructure

- It promotes standardization with concrete standardizable modelling languages

- It enables enterprise modelling in the above framework.

The strong points of the Purdue Architecture are

- Its easy to grasp presentation

- The philosophy on how it divides the views of the enterprise matches the way people think of (and organize) enterprises. This philosophy is eminently suitable for use by industrial practitioners.

The strength of the GRAI/GIM Architecture

- GRAI suggests a universally applicable approach to structure production management.

- The GRAI-GIM architecture allows an explicit modelling and design of the decisional structure of manufacturing systems, while most of the existing approaches are more information processing oriented.

\subsubsection{Further conclusions regarding the architectures}

In addition to the conclusions drawn from the individual questions, here are a few more comments:

1. The most important contribution of the Purdue Architecture is its detailed and practical proposal as to how to go about integrating enterprises. Some aspects (like manufacturing control) are dealt with in great detail.

2. The model and description categories of the Purdue architecture are defined in a finer detail than in the other two architectures (see the answers to Question 17). Furthermore there are detailed methods for producing all these descriptions provided one has selected a specific modelling method (or English) to capture these descriptions. 
3. The important CIMOSA contribution is to define a generic integration infrastructure (IIS) although it is not known to the authors at the moment how available the LOTOS specification is for IIS.

4. Although IIS depends on yet to be fixed international standards it was announced that prototypical implementations are already underway. The nature of the IIS's dependence is therefore not more than the dependence on any other type of operating system with communication and multiprocessing facilities. In fact it is only anticipated that the IIS will have an ODP implementation.

5. The other important CIMOSA contribution is the definition of its modelling framework. With some amendments (to be treated in conjunctions with the Matrix Method [see Chapter 17]) it might be usable with Purdue and other methodologies.

6. A possibility not yet explored of the CIMOSA modelling framework is its application to the integration process itself. This would enable the use of a great number of software technology results and make it possible to apply, in conjunction with CIMOSA or the other methodologies, software process models/standards.

7. The GRAI-GIM architecture is structurally closer to the Purdue architecture than CIMOSA in the sense that it changes the number of views/ aspects after the detailed design models (This change is called 'user oriented' vs. 'technical' division in GRAI-GIM terminology).

8. The GRAI-GIM modelling framework is very close to CIMOSA, with the exception that GRAI-GIM uses basically standard methods while CIMOSA uses a single tailor made one. GRAI-GIM architectural models are therefore easier to support with a diversity of CASE tools.

9. We believe that the number of viewpoints (aspects) as defined in CIMOSA is bound to expand in the future and that the development of matching (CIMOSA compliant) modelling languages instead of existing ones may prove to be a bottleneck. Note that modelling languages take $10-15$ years to mature and there is no shortcut known.

10.The CIMOSA approach gains however, an easier coordination of the different aspects as more formal cross model checking is made possible.

11. A major lack of every architecture studied is that they put forward life cycle models which involve only those processes which directly contribute to the transformation of various forms and levels of enterprise descriptions. The life cycle models need to be expanded to include models of the 'transition from computer supported business to the information integrated enterprise.' Many processes necessary to this transition would then get represented and coordinated with the rest even though 
they might not directly contribute to the transformation of architecture documents.

\subsubsection{Vision of the future, for further study}

We foresee the following tasks ahead:

1. Map the architectures onto one another in order to identify in detail the correspondence between the processes and models described by each.

2. Prepare case studies to apply CIMOSA, GRAI-GIM and Purdue to the same (kind of) project in order to compare the results. Also a case study to use the Purdue Implementation Procedures Manual but with CIMOSA and GRAI models could validate or disprove the conjecture that these combinations could significantly advance the state-of-the-art.

3. Produce a generic model for the 'transition from the computer supported business to the information integrated enterprise' as a supplement to a pure life cycle model.

4. Evaluate the use of emerging techniques as possible components of the IIS (like distributed databases, heterogeneous databases, multimedia communication, distributed AI, etc.)

5. Develop a combined architecture, possibly consisting of the generic enterprise integration process (a la Purdue) and the descriptions produced using the combined CIMOSA and GRAI model categories.

6. The most detailed correspondence between the candidate architectures could be unveiled by developing the respective meta models for these architectures. 